\section{Fosinopril decreases levels of soluble vascular cell adhesion molecule-1 in Type II diabetic patients suffering from microalbuminuria}

\begin{abstract}
Dear Sir,
Angiotensin converting enzyme inhibitors (ACE-I) have become a mainstay for the treatment of heart failure and of diabetic microalbuminuria. ACE-I have recently been found to decrease plasma levels of the vascular cell adhesion molecule1 (VCAM-1) in patients suffering from congestive heart failure [1]. The expression of VCAM-1, intercellular adhesion molecule-1 (ICAM-1) and E-selectin is preferentially found at inflammatory sites such as atherosclerotic lesions, where they mediate the recruitment of monocytes into the plaque. As activated endothelial cells release adhesion molecules, serum concentrations of circulating adhesion molecules (cAM) appear to reflect endothelial activation in-vivo. Hence, increased cAM levels are found in atherosclerosis [2] and diabetes mellitus [3]. As elevated cVCAM-1 levels are characteristic of patients suffering from diabetic microalbuminuria [4], we were interested whether the lowering effects of ACE-I on plasma levels of cVCAM-1 in heart failure could be reproduced in microalbuminuric Type II diabetic patients.

We enrolled eleven patients who had been suffering from diabetes Type II for $14 \pm 8$ years (m/f ratio: $6 / 5$, aged $54 \pm 8$ years) and were receiving insulin treatment $(n=4)$, oral antidiabetics $(n=4)$, or being managed with dietary measures only $(n=3)$. Inclusion criteria were microalbuminuria in excess of $200 \mathrm{mg} /$ day and exclusion criteria were clinically manifest heart failure (NYHA III-IV) or glucocorticoid treatment. Healthy subjects (m/f ratio: $37 / 45$; aged $54.4 \pm 9.0$ years) served as control subjects. After a baseline blood sample was drawn all patients were treated for 12 weeks with $10 \mathrm{mg}$ fosinopril qd. All soluble adhesion molecules were determined by enzyme immunoassays for soluble E-selectin, ICAM-1 and VCAM-1 (R\&D Systems, Oxon, OX14 3 YS, UK) as described previously [5]. Changes in measured endpoints after initiation of fosinopril treatment were compared with baseline
\end{abstract}

Corresponding author: S. Gasic, M. D., Department of Internal Medicine III, Division of Endocrinology \& Metabolism, University Hospital of Vienna, Währinger Gürtel 18-20, A-1090 Wien, Austria values using the Wilcoxon signed ranks test. Comparisons between patients and healthy control subjects were made with the Mann Whitney U-test.

Baseline blood glucose levels averaged $143 \pm 45$ and $87 \pm 13 \mathrm{mg} / \mathrm{dl}$ and mean $\mathrm{HbA}_{1 \mathrm{c}}$ was $8.0 \% \pm 1.1$ and $5.5 \% \pm 0.2$ in patients and control subjects, respectively $(p<0.01)$, and were not affected by fosinopril treatment. However, microalbuminuria decreased by $-44 \%$ (CI: -65 to $-22 \%$; $p=0.004$; Fig. 1). Baseline systolic and diastolic blood pressure averaged $140 \pm 13$ and $81 \pm 7 \mathrm{~mm} \mathrm{Hg}$ and fell by $-6 \%$ (-14 to $8 \%)$ and $-4 \%(-16$ to $12 \% ; p>0.05)$ in response to fosinopril. Plasma concentrations of cE-selectin, cICAM-1 and cVCAM1 were markedly higher in patients than in control subjects $(p<0.001$; Fig. 1$)$. Serum levels of cVCAM-1 decreased by $-19 \%$ (CI: -25 to $-13 \%$ ) after 12 weeks of treatment with fosinopril (Fig. 1; $p=0.003$ ) and were no longer different from those of the control group, whereas cE-selectin and cICAM-1 remained unaltered.

Thus, twelve weeks of fosinopril treatment reduced cVCAM-1 levels by $19 \%$ to concentrations seen in healthy subjects. This correlates with the report that ACE-I decreased cVCAM- 1 by about $20 \%$ in patients suffering from congestive heart failure [1]. In that trial only a subgroup of patients, in whom ACE-I increased the acetylcholine induced forearm blood flow, responded with a decrease in cVCAM-1. This supports the concept that enhancement of endothelium dependent relaxation by ACE-I was associated with the decrease in cVCAM-1. Inhibition of ACE (kininase II) increases the concentration of bradykinin, which stimulates nitric oxide (NO) production. Nitric oxide in turn down-regulates the expression of VCAM-1 [6]. Whether an ACE-I induced increase in NO production contributes to the decrease in cVCAM-1 in patients suffering from microalbuminuria, however, remains to be determined.

An alternative effector pathway explaining the observed decrease in cVCAM-1 would be reduced generation of angiotensin II, which, however, had no acute effects on cAM levels when infused at doses that increased mean arterial blood pressure by $20 \%$ [7]. The observed ACE-I induced decrease in cVCAM-1 may be of clinical relevance, because cVCAM-1 is elevated by $20 \%$ to $30 \%$ in atherosclerosis [2], and appears to correlate with the extent of atherosclerosis [8]. Thus, any decrease in cVCAM-1 may be beneficial with regard to the cardiovascular system. Secondly, cVCAM-1 is also increased in diabetic patients [4] and appears to be characteristic of microangiopathy $[9,10]$. Thirdly, evidence is growing that soluble cE-selectin and cVCAM-1 induce angiogenesis, and that their 

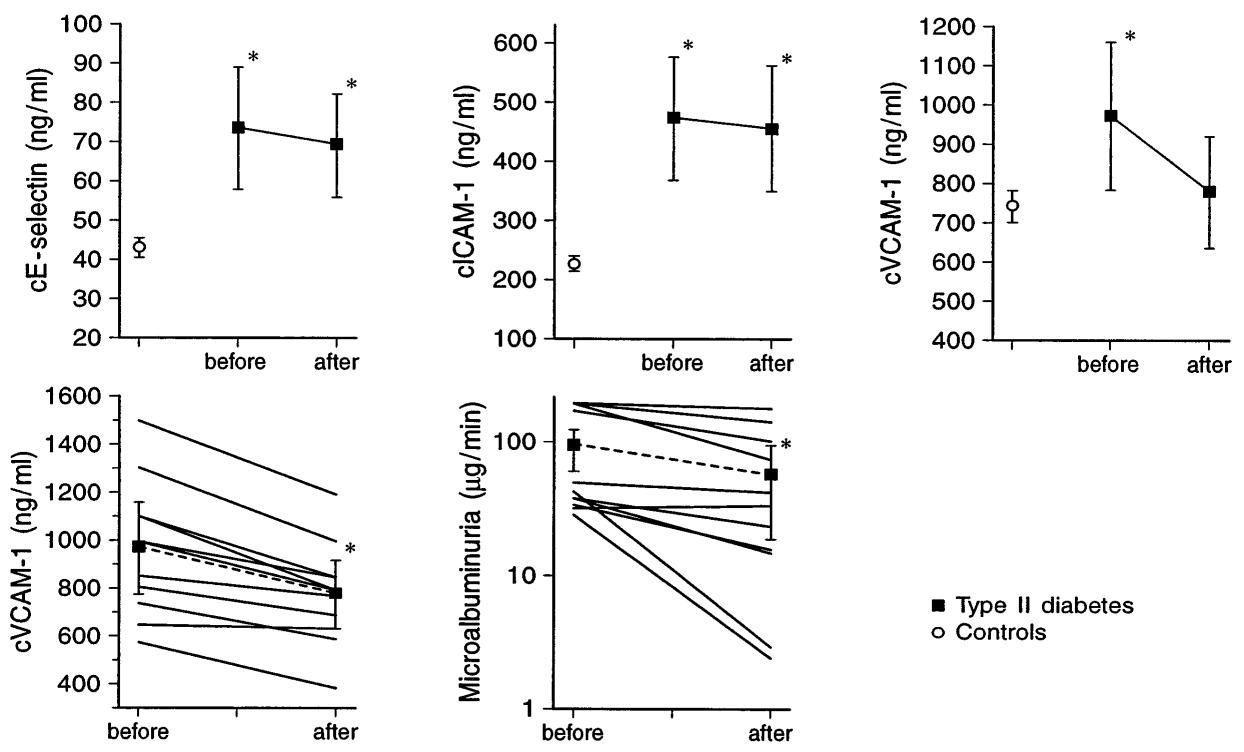

o Controls

Fig. 1. Before and after fosinopril treatment. Top layer: Comparison of serum concentrations of circulating E-selectin (left), intercellular adhesion molecule (cICAM-1; middle) and vascular cell adhesion molecule (cVCAM-1; right) in eleven patients (ם) suffering from microalbuminuria due to Type II diabetes before and after 12 weeks of fosinopril treatment $(10 \mathrm{mg} /$ day $)$ with serum concentrations measured in untreated healthy control subjects $(O ; n=82)$. Bottom layer: Individual changes in cVCAM-1 and microalbuminuria in the Type II diabetic patients

$* p<0.005$ vs control subjects (top layer), and $p<0.005$ vs pretreatment values (lower layer)

plasma levels are related to the presence [4] and the degree of diabetic retinopathy [10]. The latter study also showed that antibodies against either cAM partially inhibit retinal endothelial cell migration in response to diabetic serum. Finally, renal VCAM-1 expression also seems to play a role in the pathogenesis and propagation of a variety of kidney diseases [11] so that it is tempting to speculate that elevated VCAM-1 may contribute to the development of microalbuminuria.

As cE-selectin appears to be under direct metabolic control in Type II diabetes [12], the failure of ACE-I to affect plasma glucose or $\mathrm{HbA}_{1 \mathrm{c}}$ may explain unchanged cE-selectin levels.

In conclusion, ACE-I therapy lowered cVCAM-1 levels along with microalbuminuria and may represent a new mechanism to reduce diabetes associated endothelial dysfunction, although a comparison with other antihypertensive drugs is needed to clarify the specificity of the effect for ACE-I. Whether decreased VCAM-1 expression is directly responsible for the observed reduction in microalbuminuria remains to be elucidated.

Yours sincerely,

S. Gasic, O. F. Wagner, P. Fasching, C. Ludwig, M. Veitl, S. Kapiotis, B. Jilma

\section{References}

1. Drexler H, Kurz S, Jeserich M, Munzel T, Hornig B (1995) Effect of chronic angiotensin-converting enzyme inhibition on endothelial function in patients with chronic heart failure. Am J Cardiol 76: 13E-18E

2. Blann AD, McCollum CN (1994) Circulating endothelial cell/leukocyte adhesion molecules in atherosclerosis. Thromb Haemost 72: 151-154

3. Wagner OF, Jilma B (1997) Putative role of adhesion molecules in metabolic disorders. Horm Metab Res 29: 1-4

4. Fasching P, Veitl M, Rohac M et al. (1996) Elevated concentrations of circulating adhesion molecules and their association with microvascular complications in insulin-dependent diabetes mellitus. J Clin Endocrinol Metab 81: 4313-4317

5. Jilma B, Eichler HG, Breiteneder H et al. (1994) Effects of 17 beta-estradiol on circulating adhesion molecules. J Clin Endocrinol Metab 79: 1619-1624

6. Tsao PS, Buitrago R, Chan JR, Cooke JP (1996) Fluid flow inhibits endothelial adhesiveness. Nitric oxide and transcriptional regulation of VCAM-1. Circulation 94: 1682-1689

7. Krejcy K, Eichler HG, Jilma B et al. (1996) Influence of angiotensin II on circulating adhesion molecules and blood leukocyte count in vivo. Can J Physiol Pharmacol 74: 9-14

8. Peter K, Nawroth P, Conradt C et al. (1997) Circulating vascular cell adhesion molecule-1 correlates with the extent of human atherosclerosis in contrast to circulating intercellular adhesion molecule-1, E-selectin, P-selectin, and thrombomodulin. Arterioscler Thromb Vasc Biol 17: 505-512

9. Fasching P, Waldhäusl W, Wagner OF (1996) Elevated circulating adhesion molecules in NIDDM - potential mediators in diabetic macroangiopathy [letter]. Diabetologia 39: 1242-1244

10. Olson JA, Whitelaw CM, McHardy KC, Pearson DWM, Forrester JV (1997) Soluble leucocyte adhesion molecules in diabetic retinopathy stimulate retinal capillary endothelial cell migration. Diabetologia 40: 1166-1171

11. Rabb H, Rosen R, Ramirez G (1995) VLA-4 and its ligands: relevance to kidney diseases. Springer Semin Immunopathol 16: 417-425

12. Kautzky-Willer A, Jilma B, Fasching P, Waldhäusl W, Wagner OF (1997) Persistent elevated circulating E-selectin levels after gestational diabetes. J Clin Endocrinol Metab 82: 4117-4121 